\title{
Dynamics of Interstellar Dust Particles in Electromagnetic Radiation Fields
}

\section{Herranen, Joonas}

IEEE

2016

Herranen , J , Markkanen , J \& Muinonen , K 2016 , Dynamics of Interstellar Dust Particles in Electromagnetic Radiation Fields . in 2016 URSI INTERNATIONAL SYMPOSIUM ON ELECTROMAGNETIC THEORY (EMTS) . URSI International Symposium on Electromagnetic Theory, IEEE , New York , pp. 251-254 , URSI International Symposium on Electromagnetic Theory , Espoo, Finland, 14/08/2016 . https://doi.org/10.1109/URSI-EMTS.2016.7571366

http://hdl.handle.net/10138/228805

https://doi.org/10.1109/URSI-EMTS.2016.7571366

other

acceptedVersion

Downloaded from Helda, University of Helsinki institutional repository.

This is an electronic reprint of the original article.

This reprint may differ from the original in pagination and typographic detail.

Please cite the original version. 


\title{
Dynamics of Interstellar Dust Particles in Electromagnetic Radiation Fields
}

\author{
Joonas Herranen*, Johannes Markkanen*, and Karri Muinonen ${ }^{\dagger}$ \\ *Department of Physics, University of Helsinki, Finland \\ e-mail: joonas.herranen@ helsinki.fi \\ ${ }^{\dagger}$ Finnish Geospatial Research Institute FGI, National Land Survey, Finland
}

\begin{abstract}
We establish a theoretical framework for solving the equations of motion for an arbitrarily shaped, isotropic, and homogeneous dust particle in the presence of radiation pressure. The scattering problem involved is solved by a surface integral equation method, and a rudimentary sketch of the numerical implementation is introduced with preliminary results agreeing with predictions.
\end{abstract}

\section{INTRODUCTION}

The observed polarization of the interstellar medium is due to scattering from asymmetrical, aligned dust particles. Alignment of interstellar dust particles has been under meticulous study for the last few decades, and it has been firmly established that the dominant alignment method in many situations is by radiative torques [1]. The canonical method of studying the dynamics and in turn the alignment of interstellar particles is by considering orientation averaged equations of motion (EoMs) [2], which is a powerful method of analysis, when combined with numerical scattering software such as the Discrete Dipole Scattering (DDSCAT) software [3].

Due to the modern advancements of different scattering solutions, mainly of the integral equation methods (IEMs), a dynamical solution using the rigid body EoMs for arbitrary geometries without orientation averaging is possible with tolerable computational efforts. Surface integration methods have been used to study the forces and torques due to radiation pressure [4]. We introduce a theoretical framework for solving the dynamics of a dust particle implementing the PoggioMiller-Chan-Harrington-Wu-Tsai (PMCHWT) surface integral equation method [5] and sketch a corresponding numerical algorithm with preliminary results.

\section{TheORETICAL FRAMEWORK}

We introduce the necessary background for the development for a numerical solver of the rotational dynamics for an interstellar dust partcile interacting with an electromagnetic field. Combining the following subsections, a framework for solving the EoMs for a dust particle is obtained.

\section{A. Dynamics of a Rigid Body}

An interstellar dust particle is assumed to be a homogeneous, rigid body which obeys Newtonian mechanics. Interstellar dust is composed of highly asymmetric particles, thus the EoMs are left in their most general form. The most important physical quantity of the particle is its moment of inertia tensor, the matrix form of which for a discretized body composed of mass points is defined as

$\mathbf{I}=\left(\begin{array}{ccc}\sum_{i} m_{i}\left(y_{i}^{2}+z_{i}^{2}\right) & -\sum_{i} m_{i} x_{i} y_{i} & -\sum_{i} m_{i} x_{i} z_{i} \\ -\sum_{i} m_{i} x_{i} y_{i} & \sum_{i} m_{i}\left(x_{i}^{2}+z_{i}^{2}\right) & -\sum_{i} m_{i} y_{i} z_{i} \\ -\sum_{i} m_{i} x_{i} z_{i} & -\sum_{i} m_{i} y_{i} z_{i} & \sum_{i} m_{i}\left(x_{i}^{2}+y_{i}^{2}\right)\end{array}\right)$,

where the summations are done over all the mass points $m_{i}$ with coordinates $\left(x_{i}, y_{i}, z_{i}\right), i=1, \ldots, N$. This form of the inertia tensor is applicable to a situation, where the particle surface is discretized using a triangular mesh, illustrated in Fig. 1 .

As a real symmetric matrix the inertia matrix has an eigendecomposition $\mathbf{I}=\mathbf{Q} \mathbf{I}_{\mathbf{p}} \mathbf{Q}^{\boldsymbol{\top}}$, where $\mathbf{I}_{\mathbf{p}}=\operatorname{diag}\left(I_{1}, I_{2}, I_{3}\right)$ and $\mathbf{Q}$ is a rotation matrix. In the body frame, the rotational EoMs simplify to Euler's equations,

$$
\overrightarrow{\mathbf{N}}=\mathbf{I} \dot{\overrightarrow{\boldsymbol{\omega}}}+\overrightarrow{\boldsymbol{\omega}} \times(\mathbf{I} \overrightarrow{\boldsymbol{\omega}}),
$$

where $\overrightarrow{\mathbf{N}}$ is the total external torque, $\overrightarrow{\boldsymbol{\omega}}$ is the angular velocity vector and $(\dot{)}$ is shorthand for a time derivative. Now the rotational dynamics of the particle are described by the EoMs

$$
\begin{aligned}
\dot{\mathbf{R}} & =\mathbf{R} \boldsymbol{\Omega}^{*}, \\
\dot{\vec{\omega}} & =\mathbf{I}^{-1}(\overrightarrow{\mathbf{N}}-\overrightarrow{\boldsymbol{\omega}} \times(\mathbf{I} \overrightarrow{\boldsymbol{\omega}})),
\end{aligned}
$$

where $\mathbf{R}$ is the rotation matrix describing the orientation of the particle and $\Omega^{*}$ is an auxiliary matrix, which makes the upper equation equivalent with $\dot{\overrightarrow{\mathbf{r}}}=\overrightarrow{\boldsymbol{\omega}} \times \overrightarrow{\mathbf{r}}$, defined by

$$
\boldsymbol{\Omega}^{*}=\left(\begin{array}{ccc}
0 & -\omega_{z} & \omega_{y} \\
\omega_{z} & 0 & \omega_{x} \\
-\omega_{y} & \omega_{x} & 0
\end{array}\right) .
$$

\section{B. Electromagnetic Background}

The pressure effects of electromagnetic radiation were originally conceptualized in Kepler's observations of the tails of comets and formulated mathematically by Maxwell in 1873 [6]. A Lorentz force density, the force per unit volume, is

$$
\overrightarrow{\mathbf{f}}=\rho \overrightarrow{\mathbf{E}}+\overrightarrow{\mathbf{J}} \times \overrightarrow{\mathbf{B}}
$$

where $\rho$ is the charge of the volume element and $\overrightarrow{\mathbf{E}}, \overrightarrow{\mathbf{J}}$ and $\overrightarrow{\mathbf{B}}$ are the electric field, electric current and the magnetic field 


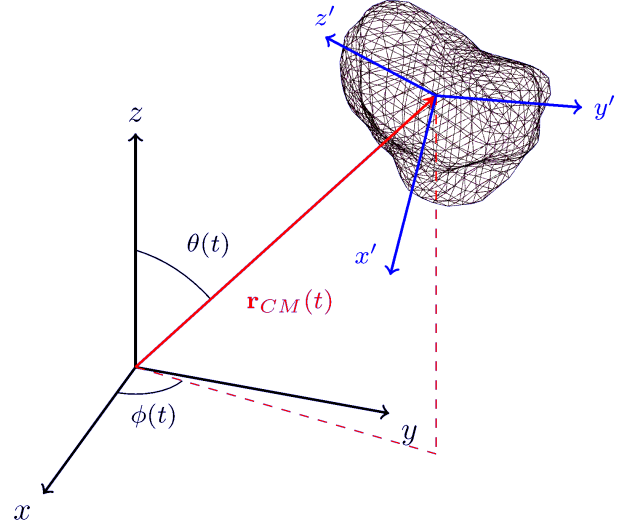

Fig. 1: A mesh discretization of a symmetric sample particle in the laboratory frame $\{x\}_{\text {lab }}$ and the body frame $\left\{x^{\prime}\right\}_{\text {body }}$.

intensity, respectively. By Maxwell equations and vector calculus identities it can be expressed as

$$
\overrightarrow{\mathbf{f}}=\nabla \cdot \mathbf{T}-\varepsilon_{0} \mu_{0} \frac{\partial \overrightarrow{\mathbf{S}}}{\partial t} .
$$

The $\overrightarrow{\mathbf{S}}$-term, where $\overrightarrow{\mathbf{S}}=\overrightarrow{\mathbf{E}} \times \overrightarrow{\mathbf{H}}$ is the Poynting vector, is the energy flux of the radiation fields, which averages to zero. The first term contains the Maxwell stress tensor with components

$$
T_{i j}=\varepsilon_{0}\left(E_{i} E_{j}-\frac{1}{2} \delta_{i j} E^{2}\right)+\frac{1}{\mu_{0}}\left(B_{i} B_{j}-\frac{1}{2} \delta_{i j} B^{2}\right),
$$

and, by integrating the force density to obtain the total force over a surface and using the divergence theorem, we obtain the total average mechanical force on the particle surface $S$,

$$
\overrightarrow{\mathbf{F}}=\oint_{S} \mathrm{~T} \cdot \hat{\mathbf{n}} \mathrm{d} S
$$

The corresponding average torque due to EM radiation is, in a straightforward fashion,

$$
\overrightarrow{\mathbf{N}}=\oint_{S} \overrightarrow{\mathbf{r}} \times(\mathrm{T} \cdot \hat{\mathbf{n}}) \mathrm{d} S .
$$

\section{The Surface Integral Equation Method}

Different methods exist for solving a scattering problem from given incident, or primary, field. When a surface mesh is considered, a surface integral equation (SIE) method will be a natural approach to the problem.

Surface integral equation formulations for linear, homogeneous, and isotropic media can be constructed in many ways, for example as the Stratton-Chu equations [7] or as an extension to the previous formulation, the PMCHWT formulation, where the total fields are expressed with respect to the primary fields $(\overrightarrow{\mathbf{E}}, \overrightarrow{\mathbf{H}})$ and equivalent electric and magnetic surface currents $\left(\overrightarrow{\mathbf{J}}_{S}, \overrightarrow{\mathbf{M}}_{S}\right)=(\hat{\mathbf{n}} \times \overrightarrow{\mathbf{H}}, \overrightarrow{\mathbf{E}} \times \hat{\mathbf{n}})$, which induce the same scattered fields as the physical scatterers [5].

The surface equivalence principle [8] states that the total fields inside the integration domain are uniquely determined by the primary fields and the equivalent surface current densities:

$$
\begin{aligned}
& \Theta(\overrightarrow{\mathbf{x}}) \overrightarrow{\mathbf{E}}(\overrightarrow{\mathbf{x}})=\overrightarrow{\mathbf{E}}^{i}(\overrightarrow{\mathbf{x}})-\frac{\eta}{\mathrm{i} k} \mathcal{L}\left[\overrightarrow{\mathbf{J}}_{S}\right]-\mathcal{K}\left[\overrightarrow{\mathbf{M}}_{S}\right], \\
& \Theta(\overrightarrow{\mathbf{x}}) \overrightarrow{\mathbf{H}}(\overrightarrow{\mathbf{x}})=\overrightarrow{\mathbf{H}}^{i}(\overrightarrow{\mathbf{x}})-\frac{1}{\mathrm{i} k \eta} \mathcal{L}\left[\overrightarrow{\mathbf{M}}_{S}\right]+\mathcal{K}\left[\overrightarrow{\mathbf{J}}_{S}\right],
\end{aligned}
$$

where $\eta=\sqrt{\frac{\mu}{\varepsilon}}$ is the impedance of the medium, $\Theta(\overrightarrow{\mathbf{x}})$ is defined for surface sources as

$$
\Theta(\overrightarrow{\mathbf{x}})=\left\{\begin{array}{l}
1, \text { if } \overrightarrow{\mathbf{x}} \in D, \text { the integration domain, } \\
1 / 2, \text { if } \overrightarrow{\mathbf{x}} \in S, \text { any surface occupying } D \\
0, \text { otherwise, }
\end{array}\right.
$$

and $\mathcal{L}$ and $\mathcal{K}$ are integrodifferential operators defined as

$$
\begin{aligned}
\mathcal{L}[\overrightarrow{\mathbf{F}}] & \equiv \int_{S_{i}} k^{2} \overrightarrow{\mathbf{F}} \phi+\nabla(\nabla \cdot \overrightarrow{\mathbf{F}}) \phi \mathrm{d} S, \\
\mathcal{K}[\overrightarrow{\mathbf{F}}] & \equiv \int_{S_{i}} \nabla \times \overrightarrow{\mathbf{F}} \phi \mathrm{d} S,
\end{aligned}
$$

where $\phi$ is the Green's function for the Helmholtz operator. A set of solvable integral equations is obtained by imposing different boundary conditions and coupling the fields in some way. The PMCHWT formulation is obtained by imposing the boundary conditions

$$
\begin{aligned}
\gamma_{t}\left(\overrightarrow{\mathbf{E}}_{1}-\overrightarrow{\mathbf{E}}_{2}\right) & =0, \\
\gamma_{t}\left(\overrightarrow{\mathbf{H}}_{1}-\overrightarrow{\mathbf{H}}_{2}\right) & =0,
\end{aligned}
$$

where $\gamma_{t}[\overrightarrow{\mathbf{F}}] \equiv-\hat{\mathbf{n}} \times \hat{\mathbf{n}} \times \overrightarrow{\mathbf{F}}$ is the tangential trace operator, and coupling the resulting electic and magnetic field integral equations with coupling constants of 1 , which results in

$$
\mathbf{A}\left(\begin{array}{c}
\overrightarrow{\mathbf{J}} \\
\overrightarrow{\mathbf{M}}
\end{array}\right)=\left(\begin{array}{c}
\gamma_{t} \overrightarrow{\mathbf{E}}^{i} \\
\gamma_{t} \overrightarrow{\mathbf{H}}^{i}
\end{array}\right)
$$

where

$$
\mathbf{A}=\left(\begin{array}{cc}
\frac{\eta_{1}}{\mathrm{i} k_{1}} \gamma_{t} \mathcal{L}_{1}+\frac{\eta_{2}}{\mathrm{i} k_{2}} \gamma_{t} \mathcal{L}_{2} & \gamma_{t} \mathcal{K}_{1}+\gamma_{t} \mathcal{K}_{2} \\
-\gamma_{t} \mathcal{K}_{1}-\gamma_{t} \mathcal{K}_{2} & \frac{1}{\mathrm{i} k_{1} \eta_{1}} \gamma_{t} \mathcal{L}_{1}+\frac{1}{\mathrm{i} k_{2} \eta_{2}} \gamma_{t} \mathcal{L}_{2}
\end{array}\right)
$$

and indices 1 and 2 separates the homogeneous and isotropic background and scatterer. [9]

\section{NUMERICAL METHODS}

A Fortran software for solving the EoMs for a surface meshed particle, assumed to be homogeneous and isotropic, has been developed. The software analyses the mesh geometry and calculates and diagonalizes the inertia matrix for the particle. The radiative torques and forces are calculated by an implementation of the direct PMCHWT formulation with Rao-Wilton-Glisson (RWG) [10] basis and testing functions [9], which makes it possible to calculate the forces and torques quickly in any particle orientation, if the primary fields are rotated in the calculations instead of the particle. This in turn makes a straightforward integration of the EoMs possible without any approximations of the particle geometry other than what is limited by the mesh. 
The problem setup is always in the laboratory frame. The EoMs are solved in the principal axes frame (principal frame) and the scattering problem must be solved in some body frame. For simplicity the body frame is chosen so that it equals the laboratory frame at $t=0$. Thus two rotation matrices, the orientation matrix $\mathbf{R}$ and the diagonalization matrix $\mathbf{Q}$, are needed to navigate between the frames. The framework is summarized in Fig. 2.

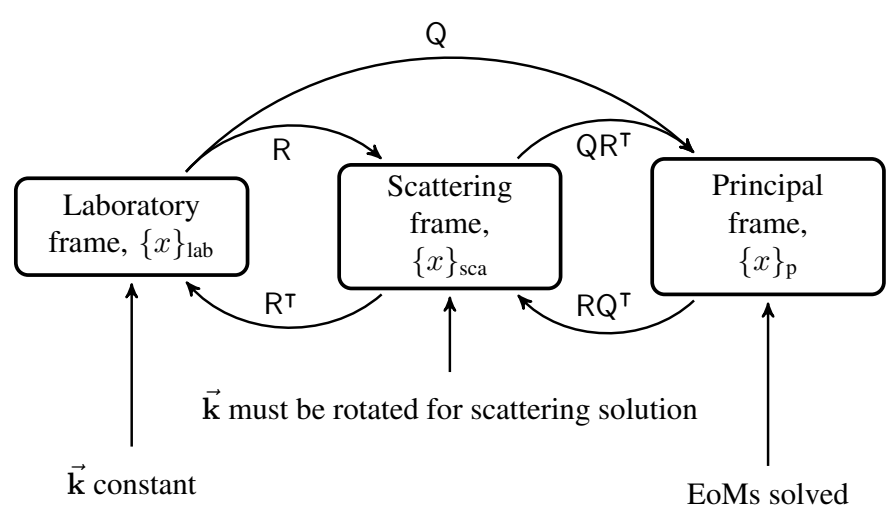

Fig. 2: Diagram of the different coordinate changes used and the significance of each frame in the integrator.

The integrator is based on the Runge-Kutta method [11], which updates the angular velocity at each time step using the torques calculated via PMCHWT method. The corresponding new rotation matrix is calculated using the Rodrigues' rotation formula [12] with the average angular velocity during a timestep. The framework of dynamics integration is presented in Fig. 3.

\begin{tabular}{|c|c|c|c|}
\hline$\frac{\text { Quantity }}{\text { Coordinates }}$ & $\overrightarrow{\mathbf{k}}$ & $\overrightarrow{\mathbf{N}}$ & $\mathbf{R}$ \\
\hline$\{x\}_{\text {lab }}$ & $\overrightarrow{\mathrm{k}}_{\text {lab }}$ constant & $t=t+\Delta t$ & $\mathbf{R}(t)=\underset{\uparrow}{\mathbf{Q}^{\top}} \mathbf{R}_{\mathbf{p}}(t)$ \\
\hline$\{x\}_{\text {sca }}$ & $\overrightarrow{\mathbf{k}}_{\mathrm{sca}}=\mathbf{R}^{\boldsymbol{\top}}(t) \overrightarrow{\mathbf{k}}_{\mathrm{lab}}$ & $\begin{array}{r}\quad \overrightarrow{\mathbf{N}}_{\mathrm{sca}}(t) \\
\quad(2)\end{array}$ & (4) \\
\hline$\{x\}_{\mathrm{p}}$ & & $\overrightarrow{\mathbf{N}}_{\mathrm{p}}(t)=\mathbf{Q R}^{\boldsymbol{\top}}(t) \overrightarrow{\mathbf{N}}_{\mathrm{sca}} \stackrel{(3)}{\longrightarrow}$ & $\mathbf{R}_{\mathrm{p}}(t)$ \\
\hline
\end{tabular}

Fig. 3: The algorithm used in the integrator in a nutshell: First, rotate the wave vector (inverse transformation compared to the particle rotation) and solve the torques using PMCHWT method; second, rotate the torque vector to the principal coordinates; third, update the EoMs in the principal coordinates; and, fourth, solve the new rotation matrix in laboratory coordinates.

\section{RESUlts}

We tested the software with two particle geometries, a sphere and a Gaussian random sample sphere (GRS) [13], visualized in Fig. 4. The particles rotated with an initial angular velocity in the laboratory frame $\overrightarrow{\boldsymbol{\omega}}_{l a b}=(0,0,1) \frac{\mathrm{rad}}{\mathrm{s}}$, with size parameter $a=10^{-7} \mathrm{~m}$ and constant density $\rho=2000 \frac{\mathrm{kg}}{\mathrm{m}^{3}}$. Adaptive timestep $\Delta t_{\max }=10^{-2} \mathrm{~s}$, amplitude of the primary
$E$-field $E_{0}=10^{-2} \frac{\mathrm{v}}{\mathrm{m}}$, and relative permittivity $\varepsilon=2+0 \mathrm{i}$ were used as input parameters. The primary field was a plane wave with wave vector $\overrightarrow{\mathbf{k}}=k \hat{\mathbf{k}}=10^{7}(0,0,1)$. The adaptivity of the time step limits the maximum rotation per time step to $0.4 \mathrm{rad}$, with maximum time step of $0.01 \mathrm{~s}$.
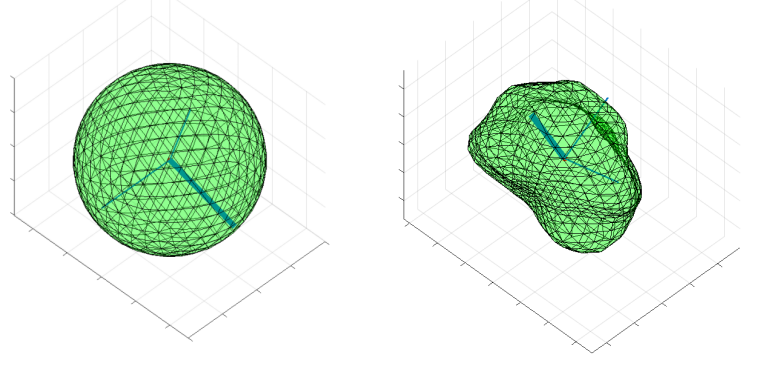

Fig. 4: The test particle geometries for the spherical and GRS particles, with their principal axes, the axis of highest principal moment highlighted.

The numerical results, summarized in Fig. 5 and Fig. 6, were consistent with expectations: the only significant force on the symmetrical sphere was in the direction of the wave vector $\overrightarrow{\mathbf{k}}$, and the GRS particle was subjected to forces in the two perpendicular directions as well. The torques on the sphere were 6 orders of magnitude smaller than on the GRS particle.
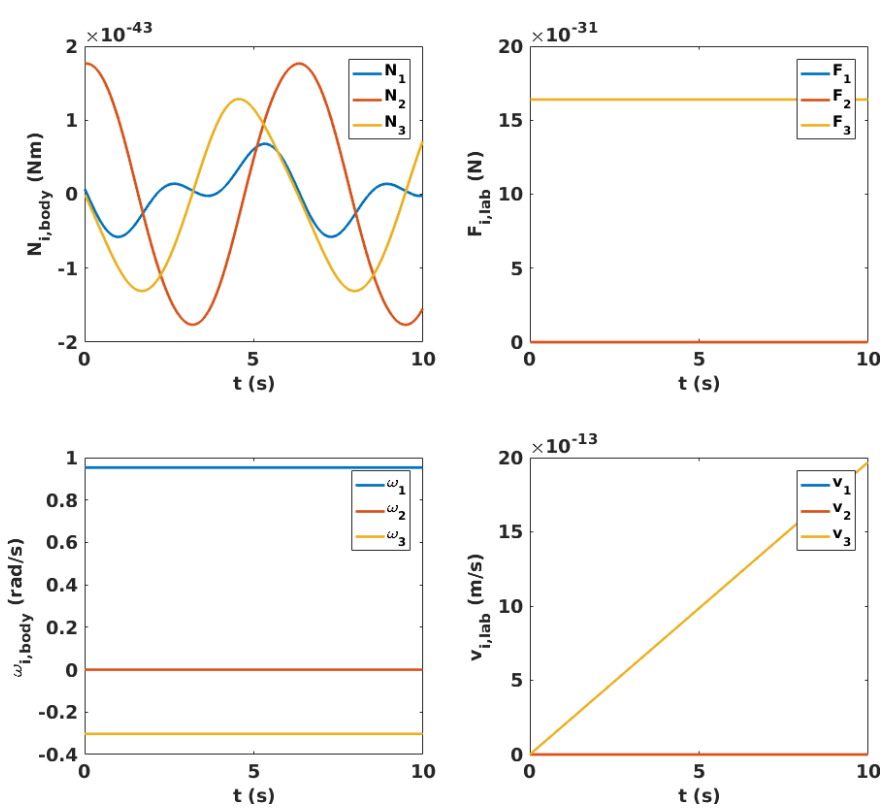

Fig. 5: Radiative torque and force induced on the sphere, calculated by the PMCHWT method and the angular velocity and linear velocities obtained by solving the EoMs. 

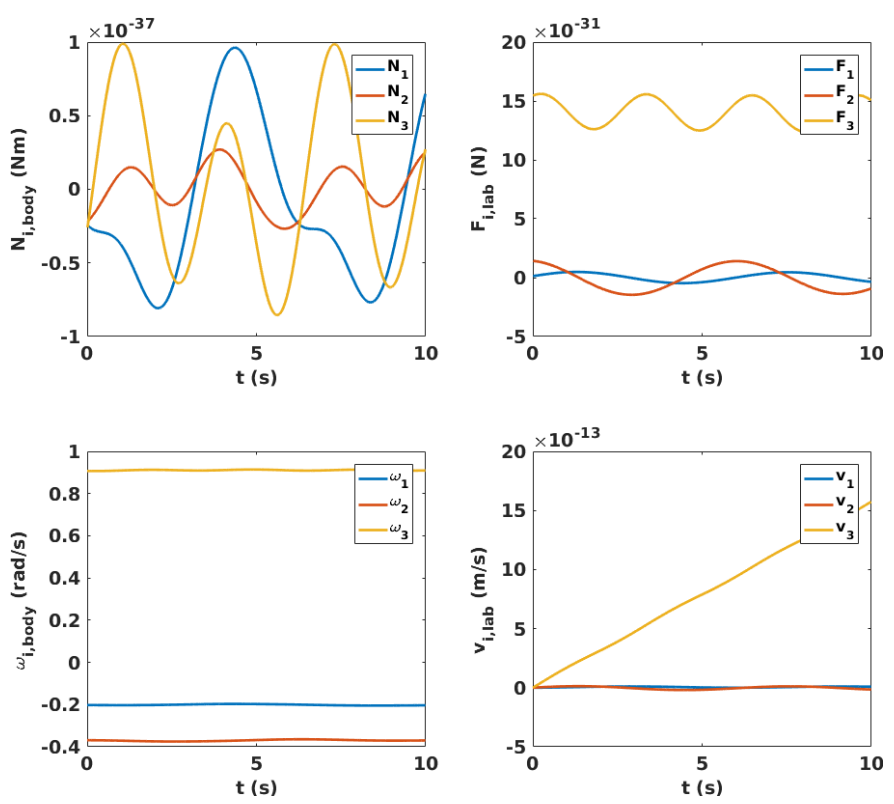

Fig. 6: As in Fig. 5 for the GRS particle. The forces and torques demonstrate the asymmetry of the GRS.

\section{CONCLUSIONS}

The direct numerical integration of rotational and linear EoMs using the PMCHWT surface integral equation method provides a novel approach to the problem of interstellar dust particle dynamics in electromagnetic radiation environment.

Results of the preliminary tests encourage further development. The main result is that the surface integral equation method provides a feasible way to solve the dynamics of rotational systems under radiation pressure explicitly.

\section{REFERENCES}

[1] A. Lazarian and T. Hoang, "Alignment of Dust by Radiative Torque: Recent Developments," 2008, arXiv: 0901.0146.

[2] B. Draine and J. Weingartner, "Radiative Torques on Interstellar Grains. II. Grain Alignment," ApJ, vol. 480, no. 2, 1997.

[3] B. Draine and P. Flatau, "Discrete-Dipole Approximation For Scattering Calculations," J. Opt. Soc. Am. A, vol. 11, pp. 1491-1499, 1994.

[4] A. Ji, T. V. Raziman, J. Butet, R. P. Sharma, and O. J. F. Martin, "Optical forces and torques on realistic plasmonic nanostructures: a surface integral approach," Opt. Lett., vol. 39, no. 16, pp. 4699-4702, 2014.

[5] A. J. Poggio and E. K. Miller, "Integral Equation Solutions of Threedimensional Scattering Problems," International Series of Monographs in Electrical Engineering, pp. 159-264, 1973.

[6] J. Maxwell, A Treatise on Electricity and Magnetism, Vol 2. Cambridge University Press, 1873, vol. 2.

[7] J. Stratton, Electromagnetic Theory. McGraw-Hill Company, 1941, vol. 1.

[8] S. Rengarajan and Y. Rahmat Samii, "The Field Equivalence Principle: Illustration of the Establishment of the Non-intuitive Null Fields," Antennas and Propagation Magazine, IEEE, vol. 42, no. 4, pp. 122 $-128,2000$.

[9] P. Ylä-Oijala, J. Markkanen, S. Järvenpää, and S. Kiminki, "Surface and Volume Integral Equation Methods for Time-harmonic Solutions of Maxwell's Equations," PIER, vol. 149, pp. 15-44, 2014.

[10] S. Rao, D. Wilton, and A. Glisson, "Electromagnetic Scattering by Surfaces of Arbitrary Shape," Antennas and Propagation, IEEE Transactions on, vol. 30, no. 3, pp. 409-418, 1982.

[11] W. H. Press, S. A. Teukolsky, William T. Vetterling, and Brian P. Flannery, Numerical Recipes. Cambridge University Press, 2007.

[12] O. Rodrigues, "Des lois géométriques qui régissent les déplacements d'un système solide dans l'espace, et de la variation des coor données provenant de ces déplacements considérés indépendamment des causes qui peuvent, les produire," J. Math. Pures Appl., vol. 5, pp. 380-440, 1840.

[13] K. Muinonen, T. Nousiainen, P. Fast, K. Lumme, and J. Peltoniemi, "Light scattering by Gaussian random particles: ray optics approximation," Journal of Quantitative Spectroscopy and Radiative Transfer, vol. 55, pp. 577-601, 1996. 